\title{
Impacts of Indigenous Trichoderma harzianum, Trichoderma viride and Pseudomonas fluorescens on Microbial Population in Soil, Plant Growth Promoting and Disease Control Potential in Soybean
}

\author{
Shyam Singh ${ }^{1 *}$, Anjeet Jangre ${ }^{2}$ and R. K. S. Tiwari ${ }^{3}$ \\ ${ }^{1}$ Department of Plant Pathology, Sant Kabir College of Agriculture and Research Station \\ (Indira Gandhi Krishi Vishwavidyalaya), Kawardha (District-Kabirdham), \\ Chhattisgarh, India \\ ${ }^{2}$ BRSM College of Agricultural Engineering and Technology and Research Station (IGKV) \\ Pandariya Road Mungeli (C.G.), India \\ ${ }^{3}$ Department of Plant Pathology, B.T.C. College of Agriculture and Research Station (Indira \\ Gandhi Krishi Vishwavidyalaya), Bilaspur, Chhattisgarh, India \\ *Corresponding author
}

A B S T R A C T

\begin{tabular}{l} 
K e y w or d s \\
Collar rot, Target \\
leaf spot, \\
Pseudomonas \\
fluorescens, \\
Soybean \\
Trichoderma \\
harzianum, \\
Trichoderma viride \\
\hline $\begin{array}{l}\text { Article Info } \\
\text { Accepted: } \\
\text { 12 January } 2021 \\
\text { Available Online: } \\
\text { 10 February } 2021\end{array}$ \\
\hline
\end{tabular}

Soybean (Glycine max L. Merrill) is an important leguminous crop contain huge amount of protein and oil. Among the fungal diseases of soybean, collar rot caused by Sclerotium rolfsii and charcoal rot caused by Corynespora cassiicola are the economically important diseases which attack on root, stem and foliate parts of the plants. An experiment was conducted on efficacy of indigenous Trichoderma harzianum, Trichoderma virideand Pseudomonas fluorescens on microbial population in soil, plant growth promoting and disease control potential in soybean and results indicated that the highest impact of Pseudomonas fluorescens was recorded on microbial population in soil which showed $26.61 \%$ increase in fungi population and $25.97 \%$ increase in bacteria population followed by $T$. viride $(18.20$ and $18.07 \%$ ) and $T$. harzianum(7.92 and $7.90 \%)$. Minimum mortality (1.97\%) was recorded with the application of $\mathrm{T}_{10}-$ Seed treatment with $\mathrm{T}_{18} @ 20 \%$ followed two foliar sprays followed by $\mathrm{T}_{9}$ - Seed treatment with $\mathrm{T}_{18} @ 15 \%$ followed two foliar sprays $(2.36 \%)$. In case target leaf spot minimum incidence $(73.54 \%)$, PDI $(10.93 \%)$ was recorded with the application of $\mathrm{T}_{14}-$ Seed treatment with Pseudomonas fluorescens @ 15\% followed $\mathrm{T}_{15}-$ Seed treatment with Pseudomonas fluorescens @ 20\% followed two foliar sprays (9.81\%). Maximum no. of pods per plant $(73.30)$, yield per plot $(4.274 \mathrm{~kg})$ and yield per ha $(27.40 \mathrm{q})$ was obtained from treatment $\mathrm{T}_{15}$ - Seed treatment with Pseudomonas fluorescens @ 20\% followed two foliar sprays followed by $\mathrm{T}_{10}-$ Seed treatment with $\mathrm{T}_{18} @ 20 \%$ followed two foliar sprays.

\section{Introduction}

Soybean (Glycine $\max$ L. Merrill) is an important leguminous crop contain huge amount of protein and oil. In India, soybean is grown in rainfed area of Madhya Pradesh, Maharashtra, Rajasthan, Chhattisgarh, Gujarat, Karnataka, Telangana, Uttar Pradesh and Uttarakhand states. Soybean has been sown on an area of 109.34 lakh in India 
during 2017-18 and total production of soybean was obtained 12.22 million tonnes during 2017-18 (Anonymous, 2018). In Chhattisgarh, soybean has grown in 1.320 lakh ha area which gives 0.863 lakh tonnes production during 2017-18 (Anonymous 2017; Sharma and Patel 2017). Both area and production of soybean has been decreased during 2017-18 as compared to 2016-17 due to the low productivity during previous years. The production and productivity of soybean are extremely influence by time and pattern of rainfall in Chhattisgarh state resulting cultivation of soybean has been discouraged in Chhattisgarh. Main reasons of low productivity are less rainfall during sowing and crop period, high rainfall at the maturity to harvesting time,attack of several diseases during floweringto maturity stage.In Chhattisgarh farmers are diverted to cultivate other crops such as pigeonpea, urdbean, mungbean etc due to the low productivity and also received very less profit from soybean crop. In Chhattisgarh, significant yield losses have been observed in soybean crop due to occurrence of major diseases such asrust (Phakopsora pachyrhizi), yellow mosaic disease (MYMV), bacterial pustule (Xanthomonas campestris pv. glycines), collarrot (Sclerotium rolfsi), target leaf spot (Corynespora cassiicola), charcoal rot (Macrophomina phaseolina) and leaf spot (Myrothecium roridum). Among them, all fungal diseases are soil borne except rust. Collar rot and charcoal rot are the economically important diseases which attack the root, stem and foliate parts of the plants. The target leaf spot disease of soybean causes by Corynespora cassiicola was first reported in 1945 (Olive et al., 1945). C. cassiicola is found on or within 530 plant species from 380 genera-including dicot, monocot, fern and cycad hosts and acts as a pathogen, saprophyte or endophyte (Smith, 2008). C. cassiicola have been reported on 68 different plant species causing infection on leaves, stem, and roots of plant (Dixon et al., 2009). Target leaf spot has become a major problem in all most of soybean growing states including Chhattisgarh (Patel, 2005).

Collar rot disease caused by Sclerotium rolfsii is one of the most destructive soil borne disease of soybean which causes 30$40 \%$ yield losses in India (Debbarma et al., 2017). Sclerotium rolfsii is a polyphagous fungus which has wide range of hosts. Collar rot disease can only be controlled with the seed and soil treatment with fungicides and biocontrol agents whereas, target leaf spot can be controlled by chemical fungicides and biocontrol agents applied as seed, soil and foliage treatments. Several finding concluded that both the diseases can be controlled with the seed and foliar application of bioagents. The application of microorganisms as agent for biocontrol of plant diseases in agriculture is now considered an important alternative to the use of chemical fungicides. Pseudomonas fluorescens have capability to effectively control fungal pathogens such as Fusarium oxysporium, Rhizoctonia bataticola and Sclerotium rolfsii (Ganesan and Gnanamanickam, 1987). All root and foliar diseases can also be minimized by the seed treatment with commercial formulation of Trichoderma harzianum or $T$. viride or Pseudomonas fluorescens (Kumar et al., 2015) and foliar application of Trichoderma harzianum or $T$. viride or Pseudomonas fluorescens are capable reduced the infection of Sclerotium rolfsii and C. cassiicola from the foliage in the field without using of recommended chemical fungicides such as Thiram or Carbendazim or Mancozeb or Tebuconazole. Besides, biocontrol measures solve the ecological and economical problem of disease and chemical fungicides in Indian agriculture and also give several eco-friendly innovative approaches for control of diseases. Inherent hazardous effects involved in conventional chemicals management coupled 
with the inclination of farmers towards organic farming (Parmar et al., 2018). Collar rot caused by Sclerotium rolfsii and target leaf spot caused by $C$. cassiicola are considered as economically important diseases of Soybean. Hence, a study was carried out on "efficacy of indigenous Trichoderma harzianum, Trichoderma virideand Pseudomonas fluorescens on microbial population in soil, plant growth promoting and disease control potential in soybean".

\section{Materials and Methods}

An experiment was conducted to find out the growth promoting activity and disease control efficiency of Trichoderma harzianum, Trichoderma virideand Pseudomonas fluorescens in Soybean at S.K. College of Agriculture and Research Station (IGKV), Kawardha (Kabirdham), Chhattisgarh. Two diseases viz., collar rot and target leaf spot which are more prevent in this region were targeted to minimize the diseases. Layout was made in Randomized Block Design (RBD) with 16 treatments viz., $\mathrm{T}_{1}-$ Seed treatment with Trichoderma harzianum @ 1\% followed two foliar sprays, $\mathrm{T}_{2}-$ Seed treatment with $T$. harzianum @ 5\% followed two foliar sprays, $\mathrm{T}_{3}$ - Seed treatment with T. harzianum @ $10 \%$ followed two foliar sprays, $\mathrm{T}_{4}-$ Seed treatment with T. harzianum @ 15\% followed two foliar sprays, $\mathrm{T}_{5}-$ Seed treatment with $T$. harzianum @20\% followed two foliar sprays, $\mathrm{T}_{6}$ - Seed treatment with Trichoderma viride @ 1\% followed two foliar sprays, $\mathrm{T}_{7}-$ Seed treatment with $T$. viride @ 5\% followed two foliar sprays, $\mathrm{T}_{8}-$ Seed treatment with $T$. viride@10\% followed two foliar sprays, $\mathrm{T}_{9}-$ Seed treatment with T. viride@ $@ 15 \%$ followed two foliar sprays, $\mathrm{T}_{10}-$ Seed treatment with T. viride @ 20\% followed two foliar sprays, $\mathrm{T}_{11}$ - Seed treatment with Pseudomonas fluorescens @ 1\% followed two foliar sprays, $\mathrm{T}_{12}-$ Seed treatment with $P$. fluorescens@5\% followed two foliar sprays,
$\mathrm{T}_{13}$ - Seed treatment with P. fluorescens @ $10 \%$ followed two foliar sprays, $\mathrm{T}_{14}-$ Seed treatment with $P$. fluorescens @ 15\% followed two foliar sprays, $\mathrm{T}_{15}-$ Seed treatment with P. fluorescens @ 20\% followed two foliar sprays and $\mathrm{T}_{16}-$ Control (Untreated) and three replication. Seeds of variety JS-335 were taken and treated with different formulation of bio-control agents @ $10 \mathrm{~g} / \mathrm{kg}$ seeds one day before sowing as per the treatment details. Treated seeds were sown in plots have net plot size of 4.0M X 3.9 $\mathrm{M}$ by maintaining the row to row distance of $30 \mathrm{~cm}$ and plant to plat $10 \mathrm{~cm}$. All the recommended agronomic practices were adopted to maintain the good canopy of the crop. In case of foliar spray, solution of biocontrol agents @10g/L of water was prepared as per treatments details. First spray was given at 30 days after sowing and second sprays at 45 days after sowing.

Soil samples were collected from each replication of all the treatment before sowing and after harvesting for measurement of microbial populations load in the experimental field. Microbial population was measured using serial dilution and spread method techniques on Potato Dextrose Agar Media. Three Petri dishes were prepared for each sample. Number of bacterial and fungal colony was counted using colony counter at 24, 48, 72 hours after incubation in each replication of all the treatments. Observations were also recorded on plant height $(\mathrm{cm})$, root length $(\mathrm{cm})$, number of branches per plant, number of pods per plant, grain yield. Number of nodules per plant was recorded at the time of flowering. Collar rot incidence was recorded at weekly intervals from seedling to maturity. The observation on occurrence of target leaf spot on soybean leaf was also recorded in all the treatments. The observation of disease severity was recorded on randomly selected five plant of each replication of each treatment. The severity of 
Target Leaf spot was recorded on soybean foliage using 0-9 scale described as $0=$ No lesions, $1=0.1-1 \%$ leaf area covered with lesion, limited only lower canopy, $2=1.1-10 \%$ leaf area cover with lesion, limited only lower canopy, $3=10.1-20 \%$ leaf area cover with lesion, limited only lower canopy. 4=20.1$30 \%$ of the leaf area covered, spread up to middle canopy, $5=30.1-40 \%$ of the leaf area covered, spread up to middle canopy,no defoliation, $6=40.1-50 \%$ leaf area covered, spread up to middle canopy, few leaves drops, $7=50.1-60 \%$ leaf area covered with lesion, spread up to upper canopy, few leaf drop, plant damage up to $30 \%, 8=60.1-70 \%$ leaf area covered with lesion, spread up to upper canopy, some leaf drop, plant damage 30 to $50 \%, 9=$ More than $70 \%$ leaf area covered with lesion, lesion very common on whole foliage of plant, defoliation common, death of plant common, plant damage more than $70 \%$. Percent disease index (PDI) for each treatment was calculated as follows:

Percent disease index $(\mathrm{PDI})=\frac{\text { Sum of individualdisease rating }}{\text { Total no, of plant examined } \mathrm{x} \text { Maximum no, of disease rating }} \times 100$

\section{Results and Discussion}

\section{Impact on Microbial population}

Microbial population in soil have been presented in table 1 reveal that maximum bacterial population (4.96cfu X $10^{4} \mathrm{~g}_{-}{ }^{1}$ ) was recorded after harvesting in treatment $\mathrm{T}_{15}$ Seed treatment with P. fluorescens @ 20\% followed two foliar sprays followed by $\mathrm{T}_{14}$ Seed treatment with Pseudomonas fluorescens @ 15\% followed two foliar sprays (4.81cfu X $\left.10^{4} \mathrm{~g}_{-}{ }^{1}\right), \mathrm{T}_{10}-$ Seed treatment with T.viride@ $20 \%$ followed two foliar sprays (4.63cfu X $10^{4} \mathrm{~g}_{-}{ }^{1}$ ), $\mathrm{T}_{13}$ - Seed treatment with P. fluorescens @ 10\% followed two foliar sprays $\left(4.56 \mathrm{cfu} \times 10^{4} \mathrm{~g}_{-}^{1}\right), \mathrm{T}_{9}-$ Seed treatment with T. viride @ 15\% followed two foliar sprays (4.47cfu X $10^{4} \mathrm{~g}^{1}{ }^{1}$ ) whereas, bacterial population was $3.62 \mathrm{cfu} \times 10^{4} \mathrm{~g}^{1}{ }^{1}$ before sowing. In untreated plots fungi and bacteria population was recorded 2.50 and $3.69 \mathrm{cfu} X$ $10^{4} \mathrm{~g}-^{1}$, respectively after the harvesting of crop. Highest impact of Pseudomonas fluorescens was recorded microbial population in soil which showed $26.61 \%$ increase in fungi and $25.97 \%$ increase in bacteria population over before treatment followed by $T$. viride (18.20 and $18.07 \%$ ) and T. harzianum (7.92 and $7.90 \%)$ whereas, in control plot increase in fungi population was recorded only $2.40 \%$ and increase in bacteria population was $1.93 \%$ (Figure 1).

\section{Impacts on Collar rotdisease}

Data pertaining to incidence of collar rot indicated that the minimum collar rot incidence $(1.97 \%)$ was recorded in treatment $\mathrm{T}_{10=}$ Seed treatment with T. viride @ 20\% followed two foliar sprays which was at par with $\mathrm{T}_{9}=$ Seed treatment with T. viride @ 15\% followed two foliar sprays $(2.36 \%), \mathrm{T}_{5}=$ Seed treatment with T. harzianum @ 20\% followed two foliar sprays $(2.76 \%), \mathrm{T}_{8}=$ Seed treatment with T. viride@ @ 10\% followed two foliar sprays $(2.76 \%)$ and significantly lower over $\mathrm{T}_{1}$ =Seed treatment with T. harzianum @1\% followed two foliar sprays $(5.12 \%), \mathrm{T}_{2}=$ Seed treatment with T. harzianum @ 5\% followed two foliar sprays $(4.72 \%), \mathrm{T}_{3}=$ Seed treatment with T. harzianum @ 10\% followed two foliar sprays $(4.33 \%), \mathrm{T}_{4}=$ Seed treatment with $T$. harzianum @ 15\% followed two foliar sprays (3.74\%), $\mathrm{T}_{6=}$ Seed treatment with T. viride @ $1 \%$ followed two foliar sprays (3.74\%), $\mathrm{T}_{7=}$ Seed treatment with T. viride @ 5\% followed two foliar sprays $(3.35 \%), \mathrm{T}_{11=}$ Seed treatment with $P$. fluorescens @ 1\% followed two foliar sprays $(6.10 \%), \quad \mathrm{T}_{12}=$ Seed treatment with P. fluorescens @ 5\% followed two foliar sprays $(5.31 \%), \mathrm{T}_{13=}$ Seed treatment with P. fluorescens @ 10\% followed two foliar sprays $(5.12 \%), \mathrm{T}_{14=}$ Seed treatment with Pseudomonas fluorescens @ 15\% followed two foliar sprays $(4.53 \%), \mathrm{T}_{15=}$ Seed 
treatment with P. fluorescens @ 20\% followed two foliar sprays $(3.74 \%)$. In control plot, collar rot incidence was recorded highest $(6.89 \%)$.

\section{Impacts on Target Leaf spot disease}

Minimum target leaf spot disease incidence $(68.73 \%)$ in treatment $\mathrm{T}_{15=}$ Seed treatment with P. fluorescens @ 20\% followed two foliar sprays which was at par with $\mathrm{T}_{14=}$ Seed treatment with Pseudomonas fluorescens @ $15 \%$ followed two foliar sprays (73.54\%), $\mathrm{T}_{13=}$ Seed treatment with P. fluorescens @ $10 \%$ followed two foliar sprays $(77.66 \%)$, $\mathrm{T}_{10=}$ Seed treatment with T. viride @ 20\% followed two foliar sprays $(71.82 \%), \mathrm{T}_{9}=$ Seed treatment with T.viride@15\% followed two foliar sprays $(74.57 \%), \mathrm{T}_{8}=$ Seed treatment with T. viride @ 10\% followed two foliar sprays $(77.66 \%), \mathrm{T}_{5}=$ Seed treatment with $T$. harzianum @ 20\% followed two foliar sprays (73.54\%) and significantly lower over rest of the treatments (Table 2). In case of PDI, minimum PDI $(9.81 \%)$ was recorded in treatment $\mathrm{T}_{15=}$ Seed treatment with $P$. fluorescens @ 20\% followed two foliar sprays followed by $\mathrm{T}_{10=}$ Seed treatment with $T$. viride @ 20\% followed two foliar sprays (10.00\%),

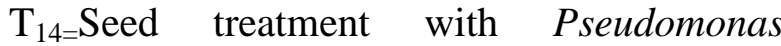
fluorescens @ 15\% followed two foliar sprays (10.93\%), $\mathrm{T}_{9}=$ Seed treatment with T.viride @ $15 \%$ followed two foliar sprays $(11.48 \%)$ and $\mathrm{T}_{13=}$ Seed treatment with $P$. fluorescens @ $10 \%$ followed two foliar sprays (12.22\%). Treatment $\mathrm{T}_{15=}$ Seed treatment with $P$. fluorescens@ 20\% followed two foliar sprays showed significantly lower PDI over $\mathrm{T}_{1}=$ Seed treatment with T. harzianum @1\% followed two foliar sprays (17.96\%), $\mathrm{T}_{2}=$ Seed treatment with T. harzianum @ 5\% followed two foliar sprays (16.11\%), $\mathrm{T}_{3=}$ Seed treatment with T.harzianum @10\% followed two foliar sprays (15.00), $\mathrm{T}_{4}=$ Seed treatment with $T$. harzianum @ 15\% followed two foliar sprays (13.70\%), $\mathrm{T}_{5}=$ Seed treatment with $T$. harzianum @ 20\% followed two foliar sprays (12.78\%), $\mathrm{T}_{6=}$ Seed treatment with T.viride @ $1 \%$ followed two foliar sprays (15.19\%), $\mathrm{T}_{7=}$ Seed treatment with T. viride @ 5\% followed two foliar sprays (14.26\%), $\mathrm{T}_{8}$ =Seed treatment with T. viride @ 10\% followed two foliar sprays (12.96\%), $\mathrm{T}_{11=S e e d ~ t r e a t m e n t}$ with P. fluorescens @ 1\% followed two foliar sprays (14.63\%) and $\mathrm{T}_{12}=$ Seed treatment with P.fluorescens @ 5\% followed two foliar sprays (13.15\%) (Table 2).

\section{Impacts on Plant growth parameters}

Maximum plant height (81.47CM) was observed in treatment $\mathrm{T}_{10=}$ Seed treatment with T.viride@20\% followed two foliar sprays. It was at par with $\mathrm{T}_{3=}$ Seed treatment with $T$. harzianum @ 10\% followed two foliar sprays (76.00CM), $\mathrm{T}_{4}=$ Seed treatment with $T$. harzianum@15\% followed two foliar sprays (77.00CM), $\mathrm{T}_{5}=$ Seed treatment with $T$. harzianum @ 20\% followed two foliar sprays (78.86CM), $\mathrm{T}_{8}=$ Seed treatment with $T$. viride @ $10 \%$ followed two foliar sprays (75.87CM), $\mathrm{T}_{9}=$ Seed treatment with $T$. viride @ $15 \%$ followed two foliar sprays (77.60CM), $\quad \mathrm{T}_{13=}$ Seed treatment with $P$. fluorescens @10\% followed two foliar sprays (76.87CM), $\quad \mathrm{T}_{14=}$ Seed treatment with Pseudomonas fluorescens @ 15\% followed two foliar sprays (78.40CM), $\quad \mathrm{T}_{15=}$ Seed treatment with P. fluorescens @ 20\% followed two foliar sprays (80.80CM) and significantly superior over rest of the treatment and minimum plant height $(72.87 \mathrm{CM})$ was recorded in untreated plot (Table 3).

In case of root length, highest root length (26.60CM) was recorded in treatment

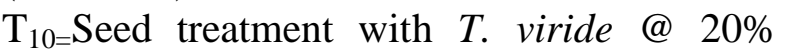
followed two foliar sprays followed by $\mathrm{T}_{15=}$ Seed treatment with $P$. fluorescens @ $20 \%$ followed two foliar sprays $(25.53 \mathrm{CM})$, 
$\mathrm{T}_{9}=$ Seed treatment with T. viride @ 15\% followed two foliar sprays (25.40CM), $\mathrm{T}_{5}$ =Seed treatment with T. harzianum @ 20\% followed two foliar sprays (25.00CM), $\mathrm{T}_{8}$ =Seed treatment with T. viride @ 10\% followed two foliar sprays (24.47CM) and $\mathrm{T}_{4}$ =Seed treatment with T. harzianum @15\% followed two foliar sprays (24.27CM) whereas, minimum root length $(25.53 \mathrm{CM})$ was observed in untreated plot (Table 3). Data pertaining to No. of primary branches per plant have been presented in table 3 reveal that the non-significant difference was recorded among the all treatments. Highest number of primary branches recorded was 5.20 per plant in $\mathrm{T}_{10=}$ Seed treatment with $T$. viride@20\% followed two foliar sprays followed by $\mathrm{T}_{9}=$ Seed treatment with $T$. viride (a) $15 \%$ followed two foliar sprays (5.07/plant) and least number of primary branches (4.41/plant) was recorded in untreated plot.

\section{Impacts on nodulation}

Data pertaining to nodulation was recorded at the time of flowering and presented in table 3 . Highest number of active nodules (43.63/plant) was recorded in treatment $\mathrm{T}_{10=}$ Seed treatment with T. viride @ 20\% followed two foliar sprays. It was at par with $\mathrm{T}_{5}=$ Seed treatment with T. harzianum @ 20\% followed two foliar sprays (40.17/plant), $\mathrm{T}_{8}$ =Seed treatment with T. viride @ 10\% followed two foliar sprays (39.67/plant), $\mathrm{T}_{9}=$ Seed treatment with T. viride @ $15 \%$ followed two foliar sprays (40.13/plant), $\mathrm{T}_{15=}$ Seed treatment with $P$. fluorescens @ $20 \%$ followed two foliar sprays (41.83/plant) and statistically significant over rest of the treatments.

\section{Impacts on Number of pods}

Maximum number of pods per plant (75.27) was recorded in treatment $\mathrm{T}_{10=}$ Seed treatment with T. viride@20\% followed two foliar sprays followed by $\mathrm{T}_{15=}$ Seed treatment with P.fluorescens @20\% followed two foliar sprays $(73.30 /$ plant $), \mathrm{T}_{9}=$ Seed treatment with T. viride@15\% followed two foliar sprays (71.67/plant), $\quad \mathrm{T}_{14=}$ Seed treatment with Pseudomonas fluorescens @ 15\% followed two foliar sprays (71.08/plant), $\mathrm{T}_{5}=$ =ed treatment with T. harzianum @ 20\% followed two foliar sprays (71.01/plant), $\mathrm{T}_{8}=$ Seed treatment with T. viride @ 10\% followed two foliar sprays (70.69/plant), $\mathrm{T}_{4}=$ Seed treatment with T. harzianum @15\% followed two foliar sprays (70.42/plant), $\mathrm{T}_{13=}$ Seed treatment with P. fluorescens @ 10\% followed two foliar sprays (70.36/plant), $\mathrm{T}_{7=}$ Seed treatment with T. viride@ @ followed two foliar sprays (69.51/plant), $\mathrm{T}_{3=}$ Seed treatment with $T$. harzianum @ 10\% followed two foliar sprays (69.38/plant), $\mathrm{T}_{12}=$ Seed treatment with $P$. fluorescens @ 5\% followed two foliar sprays (68.72/plant), $\mathrm{T}_{2}=$ Seed treatment with $T$. harzianum @ 5\% followed two foliar sprays (67.94/plant), $\mathrm{T}_{6=}$ Seed treatment with $T$. viride@1\% followed two foliar sprays

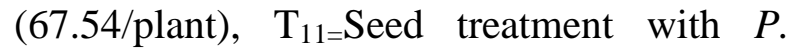
fluorescens@1\% followed two foliar sprays (66.76/plant), $\mathrm{T}_{1}=$ Seed treatment with $T$. harzianum @1\% followed two foliar sprays (66.63/plant) whereas, minimum pods per plant (65.45) was recorded in untreated plants (Table 4).

\section{Impacts on seed yield}

Seed yield per plot and per hectare have been presented in table 4 indicated that the maximum seed yield per plot $(4.388 \mathrm{~kg})$ was obtained in treatment $\mathrm{T}_{10=}$ Seed treatment with T. viride@ $20 \%$ followed two foliar sprays which was at par with $\mathrm{T}_{5}=$ seed treatment with T. harzianum @ 20\% followed two foliar sprays $(4.140 \mathrm{~kg} / \mathrm{plot}), \mathrm{T}_{8}=$ Seed treatment with T.viride@10\% followed two foliar sprays $(4.121 \mathrm{~kg} / \mathrm{plot}), \mathrm{T}_{9}=$ Seed treatment with T. viride@15\% followed two foliar 
sprays (4.178 kg/ plot), $\mathrm{T}_{14=}$ Seed treatment with Pseudomonas fluorescens @ 15\% followed two foliar sprays $(4.144 \mathrm{~kg} / \mathrm{plot})$, $\mathrm{T}_{15=}$ Seed treatment with $P$. fluorescens @ $20 \%$ followed two foliar sprays $(4.274 \mathrm{~kg} /$ plot) and significantly superior over rest all the treatments. In case of seed yield per hectare, maximum seed yield $(28.13 \mathrm{q} / \mathrm{h})$ was obtained in treatment $\mathrm{T}_{10=}$ Seed treatment with T.viride@20\% followed two foliar sprays. It was statistically at par with $\mathrm{T}_{15=}$ Seed treatment with P. fluorescens @ 20\% followed two foliar sprays $(27.40 \mathrm{q} / \mathrm{h})$, $\mathrm{T}_{9}=$ Seed treatment with T. viride @ $15 \%$ followed two foliar sprays $(26.78 \mathrm{q} / \mathrm{h})$, $\mathrm{T}_{14=}$ Seed treatment with Pseudomonas fluorescens@15\% followed two foliar sprays (26.56 q/h), $\mathrm{T}_{5}=$ Seed treatment with $T$. harzianum @20\% followed two foliar sprays ( $26.54 \mathrm{q} / \mathrm{h}), \mathrm{T}_{8}=$ Seed treatment with $T$. viride @ 10\% followed two foliar sprays (26.42 $\mathrm{q} / \mathrm{h}), \mathrm{T}_{4}=$ Seed treatment with T. harzianum @ $15 \%$ followed two foliar sprays $(26.32 \mathrm{q} / \mathrm{h})$, $\mathrm{T}_{13=}$ Seed treatment with P. fluorescens @ $10 \%$ followed two foliar sprays $(26.29 \mathrm{q} / \mathrm{h})$ and significantly superior over rest of the treatments. Minimum seed yield per hectare (24.46q) was obtained in untreated plots.

Table.1 Effect of Trichoderma harzianum, T. viride and Pseudomonas fluorescens on microbial population in soil

\begin{tabular}{|c|c|c|c|c|c|c|}
\hline \multirow[t]{3}{*}{ Treatment } & \multicolumn{6}{|c|}{ Microbial population (cfu $\left.\times 10^{6} \mathrm{~g}-{ }^{1}\right)$} \\
\hline & \multicolumn{2}{|c|}{$\begin{array}{l}\text { Before Treatment } \\
\text { (Before Sowing) }\end{array}$} & \multicolumn{2}{|c|}{$\begin{array}{l}\text { After Treatment } \\
\text { (After harvesting) }\end{array}$} & \multicolumn{2}{|c|}{$\begin{array}{l}\text { Increased population over } \\
\text { before treatment }(\%)\end{array}$} \\
\hline & Fungi & Bacteria & Fungi & Bacteria & Fungi & Bacteria \\
\hline $\begin{array}{l}T_{1}=\text { Seed treatment with } T . \text { harzianum @ } 1 \% \text { followed two foliar } \\
\text { sprays }\end{array}$ & 2.45 & 3.62 & 2.52 & 3.73 & 2.86 & 3.04 \\
\hline $\begin{array}{l}T_{2}=\text { Seed treatment with } T . \text { harzianum @ } 5 \% \text { followed two foliar } \\
\text { sprays }\end{array}$ & & & 2.57 & 3.80 & 4.90 & 4.97 \\
\hline $\begin{array}{l}T_{33} \text { Seed treatment with } T \text {. harzianum @ } 10 \% \text { followed two foliar } \\
\text { sprays }\end{array}$ & & & 2.64 & 3.90 & 7.76 & 7.73 \\
\hline $\begin{array}{l}T_{4}=\text { Seed treatment with } T \text {. harzianum @ } 15 \% \text { followed two foliar } \\
\text { sprays }\end{array}$ & & & 2.70 & 3.98 & 10.20 & 9.94 \\
\hline $\begin{array}{l}T_{5}=e e d \text { treatment with } T \text {. harzianum @ } 20 \% \text { followed two foliar } \\
\text { sprays }\end{array}$ & & & 2.79 & 4.12 & 13.88 & 13.81 \\
\hline $\mathrm{T}_{6=}$ Seed treatment with $T$. viride @ $1 \%$ followed two foliar sprays & & & 2.66 & 3.93 & 8.57 & 8.56 \\
\hline $\mathrm{T}_{7=}$ Seed treatment with $T$. viride @ 5\% followed two foliar sprays & & & 2.76 & 4.07 & 12.65 & 12.43 \\
\hline$T_{8}=$ Seed treatment with $T$. viride $@ 10 \%$ followed two foliar sprays & & & 2.89 & 4.27 & 17.96 & 17.96 \\
\hline$T_{9}=$ Seed treatment with $T$. viride @ $15 \%$ followed two foliar sprays & & & 3.03 & 4.47 & 23.67 & 23.48 \\
\hline 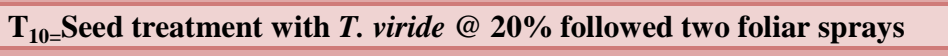 & & & 3.14 & 4.63 & 28.16 & 27.90 \\
\hline 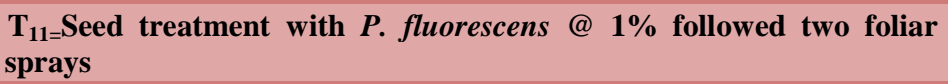 & & & 2.82 & 4.16 & 15.10 & 14.92 \\
\hline $\begin{array}{l}T_{12}=\text { Seed treatment with } P \text {. fluorescens @ 5\% followed two foliar } \\
\text { sprays }\end{array}$ & & & 2.92 & 4.31 & 19.18 & 19.06 \\
\hline $\begin{array}{l}T_{13=}=\text { Seed treatment with } P . \text { fluorescens @ } 10 \% \text { followed two foliar } \\
\text { sprays }\end{array}$ & & & 3.09 & 4.56 & 26.12 & 25.97 \\
\hline 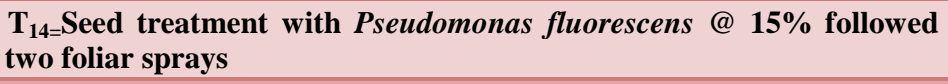 & & & 3.26 & 4.81 & 33.06 & 32.87 \\
\hline $\begin{array}{l}\mathrm{T}_{15=} \text { Seed treatment with } P . \text { fluorescens @ } 20 \% \text { followed two foliar } \\
\text { sprays }\end{array}$ & & & 3.42 & 4.96 & 39.59 & 37.02 \\
\hline$T_{16}=$ Control & & & 2.50 & 3.69 & 2.04 & 1.93 \\
\hline SEm \pm & & & 0.05 & 0.07 & - & - \\
\hline CD at $5 \%$ & & & 0.14 & 0.19 & - & - \\
\hline
\end{tabular}


Table.2 Efficacy of Trichoderma harzianum, T. viride and Pseudomonas fluorescens against collar rot and target leaf spot of soybean

\begin{tabular}{|c|c|c|c|}
\hline \multirow[t]{2}{*}{ Treatment } & \multirow{2}{*}{$\begin{array}{c}\text { Collar rot } \\
\text { incidence }(\%)\end{array}$} & \multicolumn{2}{|c|}{ Target Leaf spot } \\
\hline & & Incidence (\%) & PDI (\%) \\
\hline $\begin{array}{l}T_{1}=\text { Seed treatment with } T . \text { harzianum @ } \\
1 \% \text { followed two foliar sprays }\end{array}$ & $5.12(1308)$ & $84.54(66.85)$ & $\begin{array}{c}17.96 \\
(25.07)\end{array}$ \\
\hline $\begin{array}{l}\mathrm{T}_{2}=\text { Seed treatment with } T . \text { harzianum @ } \\
5 \% \text { followed two foliar sprays }\end{array}$ & $4.72(12.55)$ & $82.47(65.25)$ & $\begin{array}{c}16.11 \\
(23.66)\end{array}$ \\
\hline 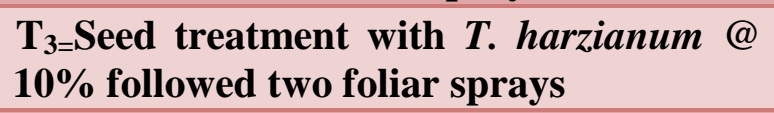 & $4.33(12.01)$ & $80.41(63.73)$ & $\begin{array}{c}15.00 \\
(22.79)\end{array}$ \\
\hline $\begin{array}{l}T_{4}=\text { Seed treatment with } T . \text { harzianum @ } \\
15 \% \text { followed two foliar sprays }\end{array}$ & $3.74(11.15)$ & $78.69(62.51)$ & $\begin{array}{c}13.70 \\
(21.72)\end{array}$ \\
\hline $\begin{array}{l}T_{5}=\text { eed treatment with } T . \text { harzianum @ } \\
20 \% \text { followed two foliar sprays }\end{array}$ & $2.76(9.56)$ & $73.54(59.04)$ & $\begin{array}{c}12.78 \\
(20.95)\end{array}$ \\
\hline $\begin{array}{l}\mathrm{T}_{6=} \text { Seed treatment with } T \text {. viride @ } 1 \% \\
\text { followed two foliar sprays }\end{array}$ & $3.74(11.15)$ & $83.51(66.04)$ & $\begin{array}{c}15.19 \\
(22.94)\end{array}$ \\
\hline 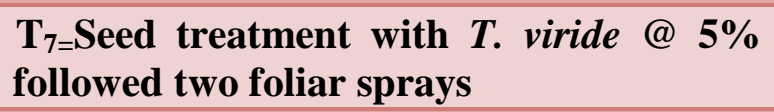 & $3.35(10.55)$ & $80.41(63.73)$ & $\begin{array}{c}14.26 \\
(22.19)\end{array}$ \\
\hline $\begin{array}{l}\mathrm{T}_{8}=\text { Seed treatment with } T . \text { viride } @ 10 \% \\
\text { followed two foliar sprays }\end{array}$ & $2.76(9.56)$ & $77.66(61.79)$ & $\begin{array}{c}12.96 \\
(21.10)\end{array}$ \\
\hline $\begin{array}{l}\mathrm{T}_{9}=\text { Seed treatment with } T . \text { viride @ } 15 \% \\
\text { followed two foliar sprays }\end{array}$ & $2.36(8.84)$ & 74.57 (59.72) & $\begin{array}{c}11.48 \\
(19.81)\end{array}$ \\
\hline 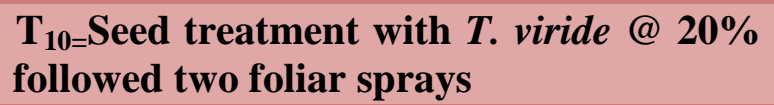 & $1.97(8.07)$ & $71.82(57.94)$ & $\begin{array}{c}10.00 \\
(18.44)\end{array}$ \\
\hline 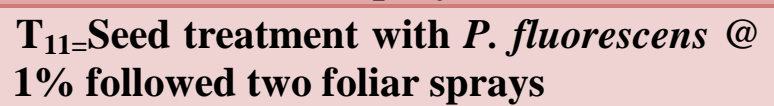 & $6.10(14.30)$ & $78.69(62.51)$ & $\begin{array}{c}14.63 \\
(22.49)\end{array}$ \\
\hline $\begin{array}{l}\mathrm{T}_{12}=\text { Seed treatment with } P . \text { fluorescens @ } \\
5 \% \text { followed two foliar sprays }\end{array}$ & $5.31(13.32)$ & $81.44(64.48)$ & $\begin{array}{l}13.15 \\
(21.26)\end{array}$ \\
\hline 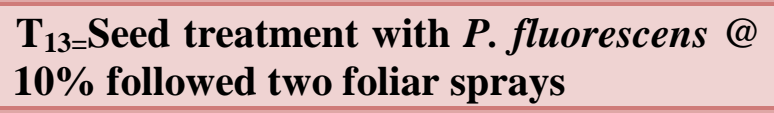 & $5.12(13.08)$ & 77.66 (61.79) & $\begin{array}{c}12.22 \\
(20.46)\end{array}$ \\
\hline $\begin{array}{l}\text { T14=Seed treatment with Pseudomonas } \\
\text { fluorescens @ } 15 \% \text { followed two foliar } \\
\text { sprays }\end{array}$ & $4.53(12.29)$ & $73.54(59.04)$ & $\begin{array}{c}10.93 \\
(19.31)\end{array}$ \\
\hline 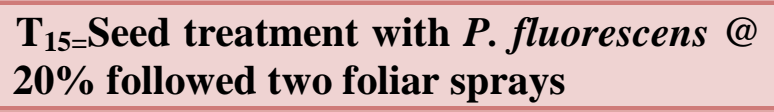 & $3.74(11.15)$ & $68.73(56.00)$ & $9.81(18.25)$ \\
\hline $\mathbf{T}_{16=}$ Control & $6.89(15.22)$ & $85.57(67.67)$ & $\begin{array}{c}19.26 \\
(26.03)\end{array}$ \\
\hline SEm \pm & 0.77 & 2.15 & 0.88 \\
\hline CD at $5 \%$ & 2.11 & 5.98 & 2.44 \\
\hline
\end{tabular}


Table.3 Effect of Trichoderma harzianum, T. viride and Pseudomonas fluorescens on plant growth and nodulation of soybean

\begin{tabular}{|c|c|c|c|c|}
\hline Treatment & $\begin{array}{l}\text { Plant } \\
\text { height } \\
\text { (cm) }\end{array}$ & $\begin{array}{l}\text { Root } \\
\text { length } \\
(\mathrm{cm})\end{array}$ & $\begin{array}{c}\text { No. of } \\
\text { primary } \\
\text { branches } \\
\text { per plant }\end{array}$ & $\begin{array}{c}\text { No. of } \\
\text { nodules } \\
\text { per plant }\end{array}$ \\
\hline $\begin{array}{l}T_{1}=\text { Seed treatment with } T \text {. harzianum } \\
\text { @ } 1 \% \text { followed two foliar sprays }\end{array}$ & 73.87 & 21.13 & 4.57 & 33.20 \\
\hline $\begin{array}{l}T_{2}=\text { Seed treatment with } T \text {. harzianum } \\
\text { @ } 5 \% \text { followed two foliar sprays }\end{array}$ & 74.53 & 22.67 & 4.62 & 34.93 \\
\hline $\begin{array}{l}T_{3}=\text { Seed treatment with } T \text {. harzianum } \\
\text { @ } 10 \% \text { followed two foliar sprays }\end{array}$ & 76.00 & 23.80 & 4.72 & 35.97 \\
\hline $\begin{array}{l}T_{4}=\text { Seed treatment with } T \text {. harzianum } \\
\text { @ } 15 \% \text { followed two foliar sprays }\end{array}$ & 77.00 & 24.27 & 4.85 & 36.07 \\
\hline $\begin{array}{l}T_{5}=\text { Seed treatment with } T \text {. harzianum } \\
\text { @ } 20 \% \text { followed two foliar sprays }\end{array}$ & 78.67 & 25.00 & 4.98 & 40.17 \\
\hline 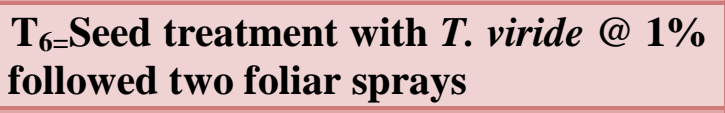 & 74.13 & 22.87 & 4.68 & 35.50 \\
\hline 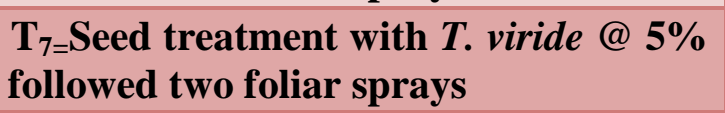 & 74.40 & 23.80 & 4.76 & 37.40 \\
\hline $\begin{array}{l}T_{8}=\text { Seed treatment with } T \text {. viride } @ \\
10 \% \text { followed two foliar sprays }\end{array}$ & 75.87 & 24.47 & 4.90 & 39.67 \\
\hline $\begin{array}{l}T_{9}=\text { Seed treatment with } T \text {. viride } @ \\
15 \% \text { followed two foliar sprays }\end{array}$ & 77.60 & 25.40 & 5.07 & 40.13 \\
\hline 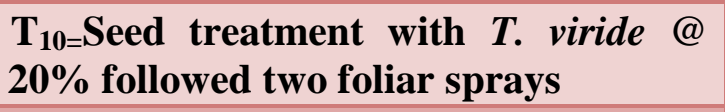 & 81.47 & 26.60 & 5.20 & 43.63 \\
\hline 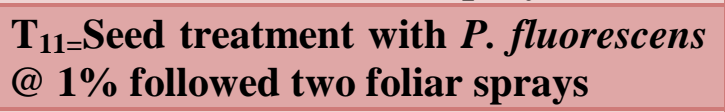 & 73.47 & 22.73 & 4.53 & 34.73 \\
\hline $\begin{array}{l}T_{12}=\text { Seed treatment with } P \text {. fluorescens } \\
\text { @ 5\% followed two foliar sprays }\end{array}$ & 74.86 & 23.33 & 4.57 & 36.70 \\
\hline 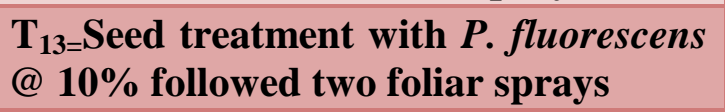 & 76.87 & 23.80 & 4.62 & 38.53 \\
\hline $\begin{array}{l}\mathrm{T}_{14=S e e d} \text { treatment with Pseudomonas } \\
\text { fluorescens @ } 15 \% \text { followed two foliar } \\
\text { sprays }\end{array}$ & 78.40 & 23.93 & 4.68 & 39.25 \\
\hline 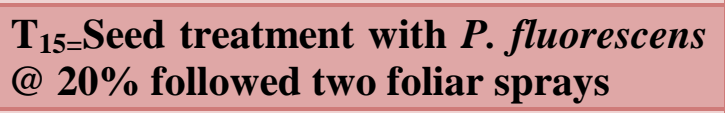 & 80.80 & 25.53 & 4.79 & 41.83 \\
\hline $\mathbf{T}_{16=}$ Control & 72.87 & 21.33 & 4.41 & 32.57 \\
\hline SEm \pm & 2.13 & 0.95 & 0.62 & 1.57 \\
\hline CD at $5 \%$ & 5.93 & 2.64 & NS & 4.37 \\
\hline
\end{tabular}


Table.4 Effect of Trichoderma harzianum, T. viride and Pseudomonas fluorescens on no. of pods and seed yield of soybean

\begin{tabular}{|c|c|c|c|}
\hline \multirow[t]{2}{*}{ Treatment } & \multirow{2}{*}{$\begin{array}{l}\text { No. of pods } \\
\text { per plant }\end{array}$} & \multicolumn{2}{|c|}{ Grain yield } \\
\hline & & Kg/plot & Quintal per ha. \\
\hline $\begin{array}{l}T_{1}=\text { Seed treatment with } T . \text { harzianum @ } \\
1 \% \text { followed two foliar sprays }\end{array}$ & 66.63 & 3.884 & 24.90 \\
\hline $\begin{array}{l}T_{2}=\text { Seed treatment with } T . \text { harzianum @ } \\
5 \% \text { followed two foliar sprays }\end{array}$ & 67.94 & 3.961 & 25.39 \\
\hline 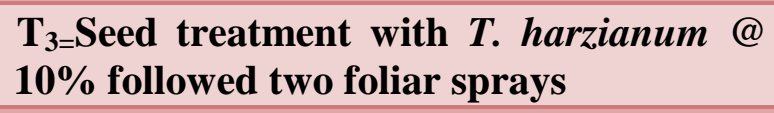 & 69.38 & 4.045 & 25.93 \\
\hline $\begin{array}{l}T_{4}=\text { Seed treatment with } T . \text { harzianum @ } \\
15 \% \text { followed two foliar sprays }\end{array}$ & 70.42 & 4.106 & 26.32 \\
\hline $\begin{array}{l}\mathrm{T}_{5}=\text { Seed treatment with } T . \text { harzianum @ } \\
20 \% \text { followed two foliar sprays }\end{array}$ & 71.01 & 4.140 & 26.54 \\
\hline 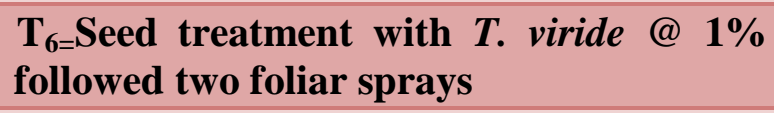 & 67.54 & 3.938 & 25.24 \\
\hline $\begin{array}{l}\mathbf{T}_{7=} \text { Seed treatment with } T \text {. viride @ } 5 \% \\
\text { followed two foliar sprays }\end{array}$ & 69.51 & 4.052 & 25.98 \\
\hline $\begin{array}{l}T_{8}=\text { Seed treatment with } T \text {. viride @ } 10 \% \\
\text { followed two foliar sprays }\end{array}$ & 70.69 & 4.121 & 26.42 \\
\hline $\begin{array}{l}T_{9}=\text { Seed treatment with } T \text {. viride @ } 15 \% \\
\text { followed two foliar sprays }\end{array}$ & 71.67 & 4.178 & 26.78 \\
\hline 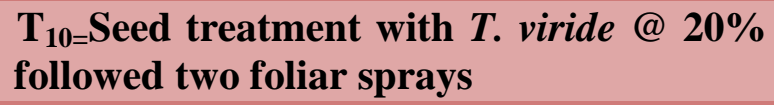 & 75.27 & 4.388 & 28.13 \\
\hline 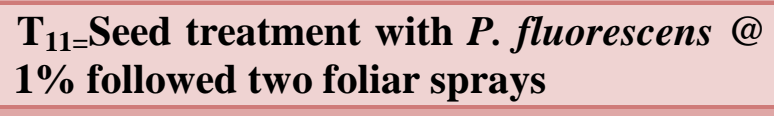 & 66.76 & 3.892 & 24.95 \\
\hline $\begin{array}{l}T_{12}=\text { Seed treatment with } P . \text { fluorescens @ } \\
5 \% \text { followed two foliar sprays }\end{array}$ & 68.72 & 4.007 & 25.68 \\
\hline 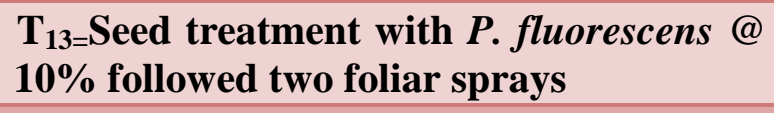 & 70.36 & 4.102 & 26.29 \\
\hline $\begin{array}{l}\text { T14=Seed treatment with Pseudomonas } \\
\text { fluorescens @ } 15 \% \text { followed two foliar } \\
\text { sprays }\end{array}$ & 71.08 & 4.144 & 26.56 \\
\hline 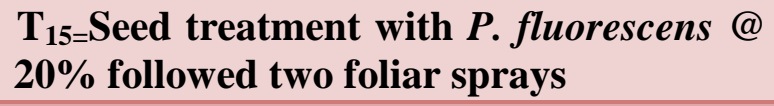 & 73.30 & 4.274 & 27.40 \\
\hline $\mathbf{T}_{16}=$ Control & 65.45 & 3.816 & 24.46 \\
\hline SEm \pm & 2.03 & 0.101 & 0.75 \\
\hline CD at $5 \%$ & 5.64 & 0.281 & 2.09 \\
\hline
\end{tabular}


Fig.1 Impact of Trichoderma harzianum, T. viride and Pseudomonas fluorescens on fungi and bacteria population

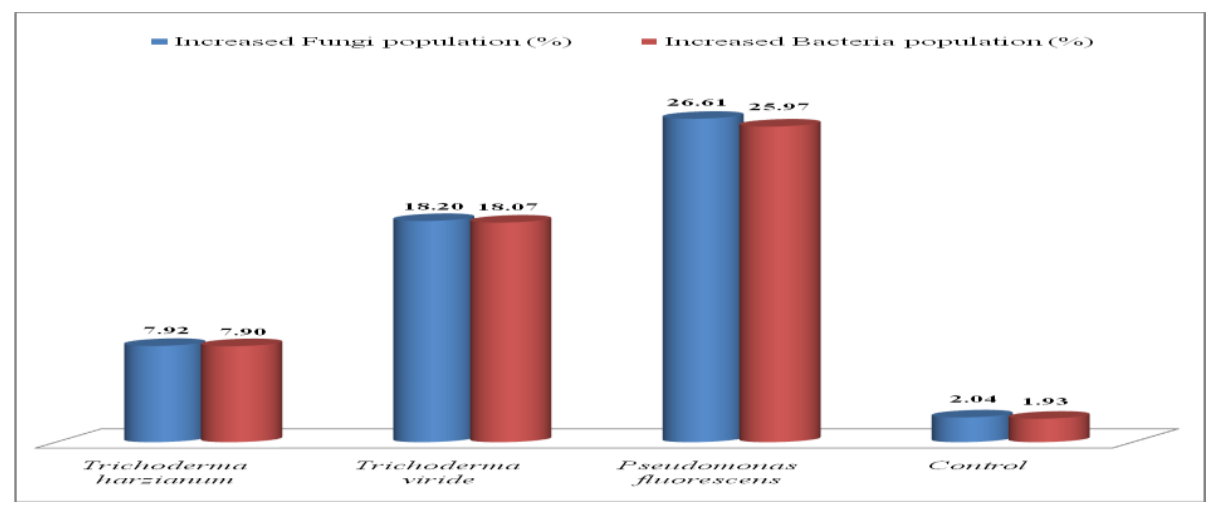

Plate.1 Impact of Trichoderma harzianum, T. viride and Pseudomonas fluorescens on soybean
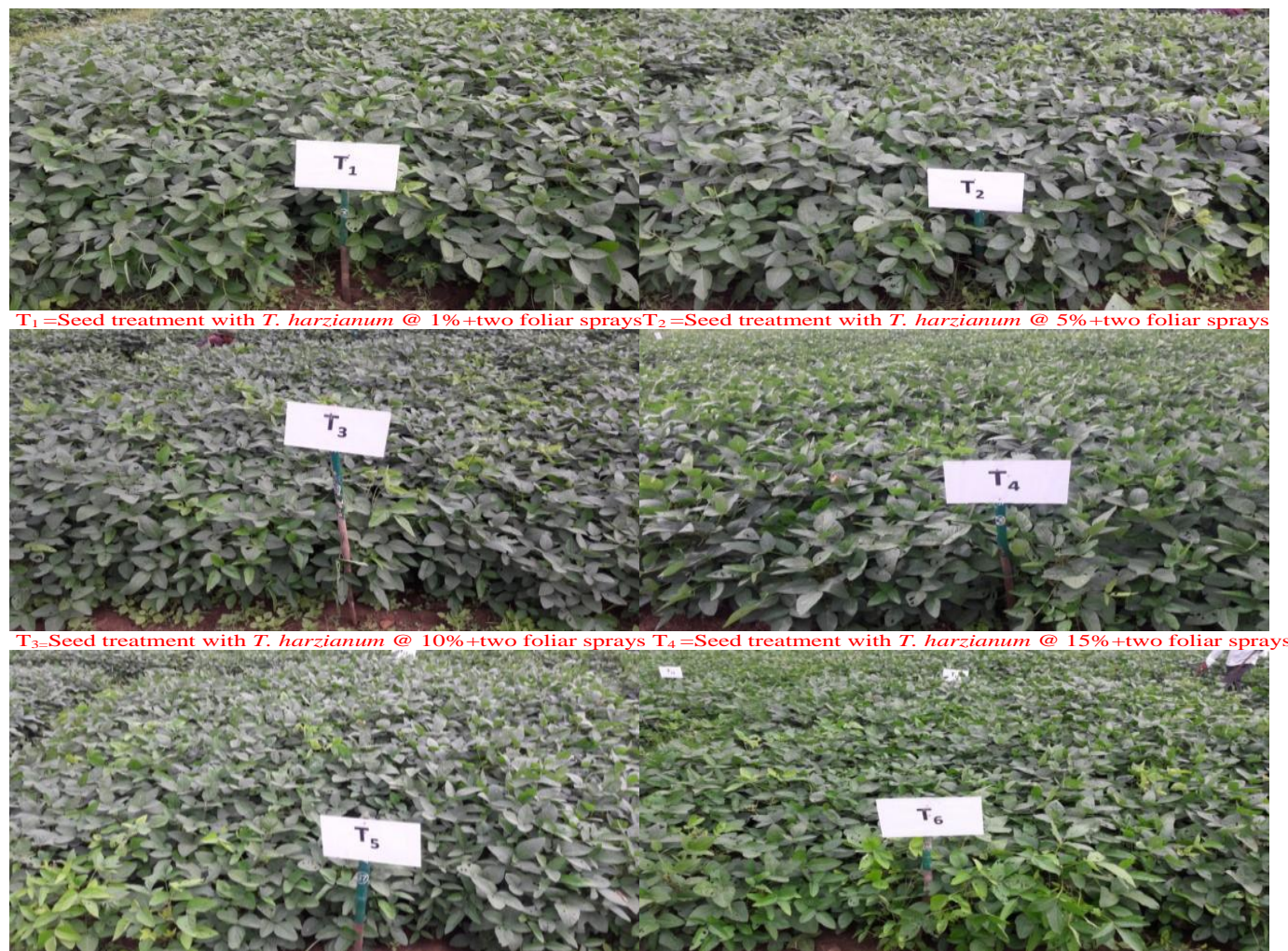

$\mathrm{T}_{5}=$ eed treatment with $T$. harzianum @20\% followed two foliar sprays $\mathrm{T}_{6=}$ Seed treatment with T.viride@1\% followed two foliar sprays

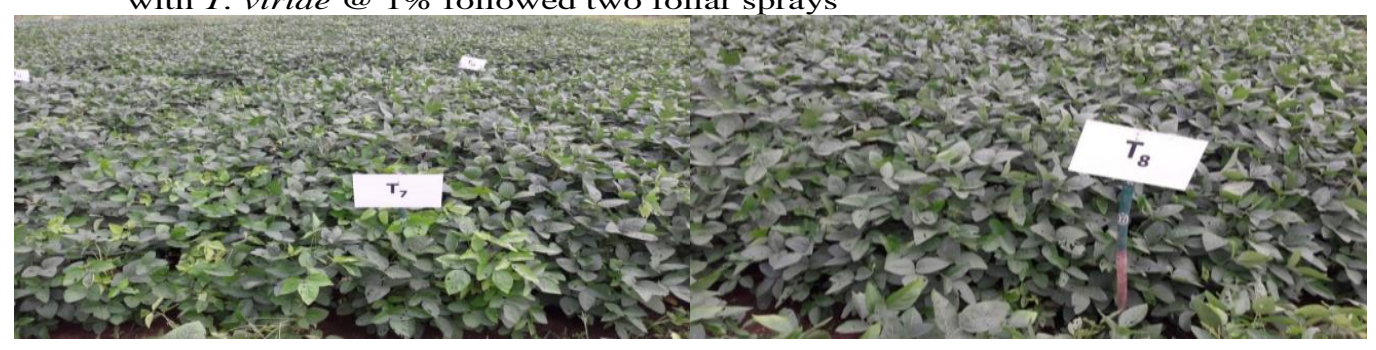


Plate.2 Impact of Trichoderma harzianum, T. viride and Pseudomonas fluorescens on soybean
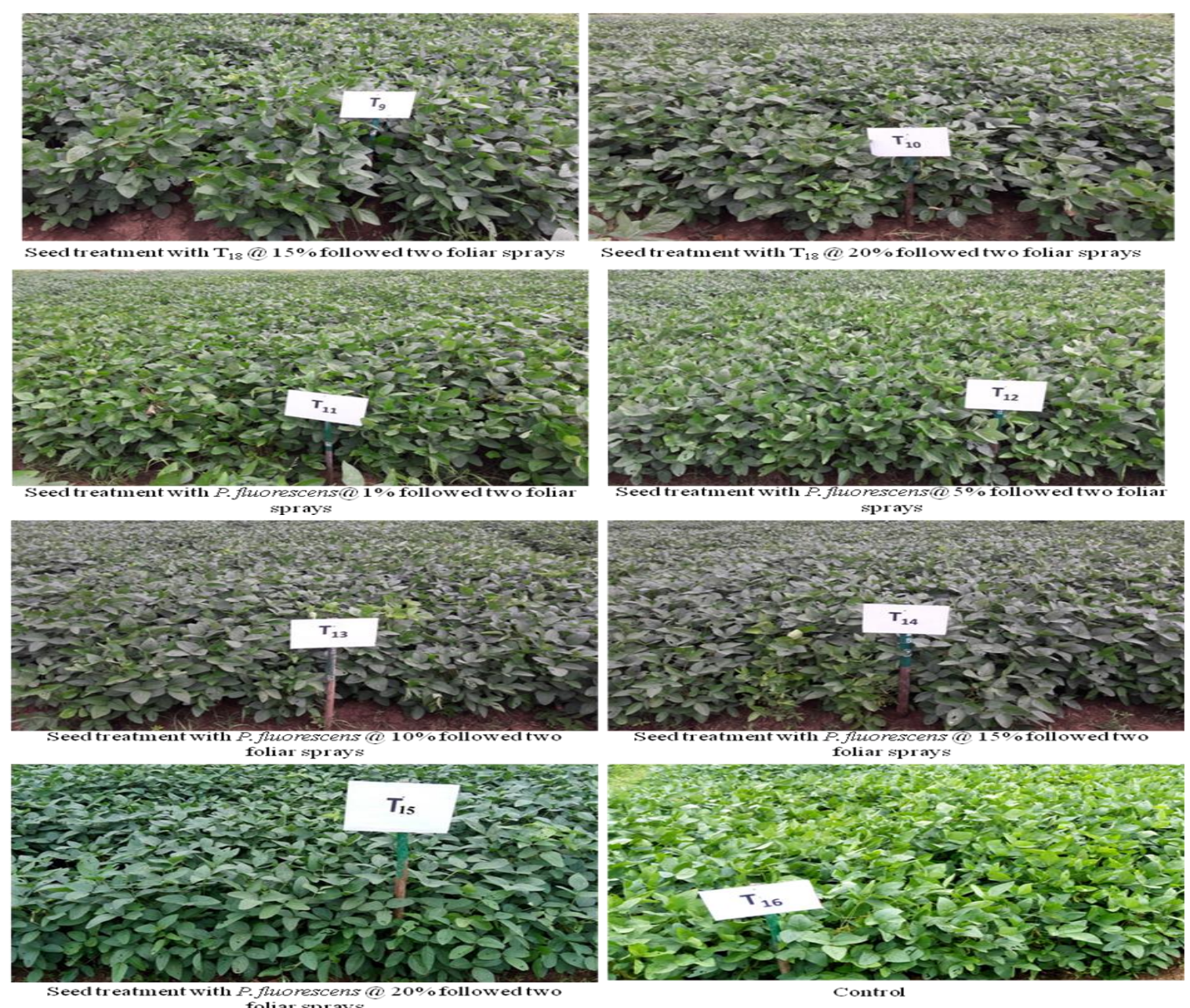

The present findings indicated that the seed treatment with $T$. virideor $T$. harzianum followed by foliar spraysshowed significant positive effects in reducing collar rot disease in soybean and also increased the plant height, root length, nodulation, number of pods and seed yield. These results corroborate with the findings of Meher et al., (2018). They tested bio-efficacy of twenty native isolates of Trichoderma spp. against Sclerotium rolfsii and their effects on growth parameters of chickpea plant. Tr- 7 was found to be most effective with minimum seedling mortality of $6.67 \%$. Trichoderma spp. has wide range of mechanisms for disease control i.e, Mycoparasitism and hyphallysis,

antibiosis, competition for nutrients and space. Several species of genus Trichoderma have been identified as growth promoting agents (Rudresh et al., 2005; Jash et al., 2007; Swathi et al., 2015). Trichoderma are more capable to enhance the growth of plants and also increase the crop productionin several crops (Balasubramanian 2003). Katwasra (2002) reported that Pseudomonas fluorescens was most effective in reducing theincidence of dry root rot with $71.8 \%$ diseasecontrol. Belkar and Gade (2013) found efficacy of seed treatment with Pseudomonas fluorescens@10g / kg of seed was found effective against Rhizoctonia, Sclerotium pathogens.Konde et al., (2017) revealed that 
seed treatment with carbendazim + Trichoderma viride recorded significantly maximum seed germination $(94.44 \%)$ while highest grain yield (1808 $\left.\mathrm{kg} \mathrm{ha}^{-1}\right)$ was observed with the soil application of Trichoderma viride + Trichoderma harzianum.Khodke and Raut (2011)found effectiveness of seed treatmentand soil application of fungicides, bioagents and its combinations in increasing seed germination and reducing pre and post emergence mortality. Suryawanshi et al., (2015) studied on collar rot caused by Sclerotium rolfsiiSacc. on brinjal and revealed significantly highest mycelial growth inhibition was recorded with Bacillus megateriumand $P$. fluorescens. Rajendraprasad et al., (2017) evaluated twenty four isolates of Trichoderma harzianum and Trichoderma viride and twelve different Baccillus subtilis and Pseudomonas fluorescence. The combination of potential Trichoderma harzianum-1 and Pseudomonas fluorescence bacterial biocontrol agents also proved effective in increasing germination and to reduce pre and post emergence collar rot in the pots when inoculated with Sclerotium rolfsii. Seed treatment with Pseudomonas fluorescence-3+ soil application with Trichoderma harzianum -1) seed treatment with Trichoderma harzianum $-1+$ soil application with Pseudomonas fluorescence-3) was recorded 52.08 and 49.17 percent germination respectively when inoculated with Sclerotium rolfsii. The lowest incidence (47.92 percent) of pre emergence damping off was recorded in seed treatment with Pseudomonas fluorescence-3 + soil application with Trichoderma harzianum -1). Gandhi et al., (2017) reported that the Pseudomonas fluorescensas as soil application gave maximum disease control $(55.11 \%)$ of collar rot of sunflower and also maximum seed yield.Ingle et al., (2018) revealed that the Pseudomonas fluorescens and Trichoderma viride seed dressing with Carboxin $37.5 \%+$
Thiram 37.5\% (combi product) @ 2g/kg most effective regarding seed germination, incidence of root rot. Shyam and Tiwari (2018) evaluated efficacy of Trichoderma harzianum with integration of fungicides collar rot disease of chickpea caused by Sclerotium rolfsii in Chhattisgarh and reveal that the Trichoderma harzianum were found significantly effective for the prevention of mortality caused by $\mathrm{S}$. rolfsii in chickpea. Singh et al., (2017) evaluated integration of Trichoderma, Pseudomonas and fungicides for the control of Collar rot disease of Chickpea and reported Trichoderma harzianum @8q/ha-1 (Soil)+ Hexaconazole @ 3ml/kg-1 seed and Pseudomonas fluorescens@8q/ha-1 (Soil) + Hexaconazole @ $3 \mathrm{ml} / \mathrm{kg}-1$ seed were significantly effective against collar rot disease.

\section{References}

Anonymous 2017. Soybean production by state. The Soybean Processors Association of India. http://www.sopa.org.

Anonymous 2018. Annual Report-2017-18, Department of Agriculture, Cooperation \& Farmers Welfare, Government of India Krishi Bhawan, New Delhi-110 001.

Anonymous 2008. Director's report and summary tables of experiments (200708). Directorate of Soybean Research (DSR), Indore. pp: i-xix.

Balasubramanian, C., P. Udaysoorian,C. Prabhu and KumarGS. 2008. Enriched compost for yield and quality enhancement in sugarcane. Journal of Eco-biology. 2:173-176.

Belkar Y.K. and Gade,RM. 2013. Management of root rot and collar rot of soybean by antagonistic microorganism. J. Pl. Dis. Sci. 8(1):39-42.

Campbell, R. 1989. Biological control of microbial plant pathogens. Cambridge 
University Press, Cambridge. U.K.Pp 50-51.

Debbarma, A., D. Majumder, J.D.Kongbrailatpam andKangjam,B. 2017. Estimation of yield loss in soybean due to collar rot disease in mid hills of Meghalaya and its management. Environment and Ecology, 35(2):10811086.

Dixon, L., R. Schlub, K. Pernezny andDatnoff,L. 2009. Host specialization and phylogenetic diversity of Corynespora cassiicola. Phytopathology, 99(9):1015-27.

Gandhi, V., R.S. Taya and Kumar,A., 2017. Management of collar rot (Sclerotium rolfsiiSacc.) in sunflower (Helianthus annuus L.). Indian J. Agric. Res. 51(6):586-590.

Ganesan, P. andGnanamanickam,S.S. 1987. Biological control of Sclerotium rolfsiiSacc. In Peanut by inoculation with Pseudomonas fluorescens. Soil Biol.Biochem. 19:35-38.

Ingle, Y.V., A.V. Zope, D.L. Wasule, M.S. Dandge and Patil,P.V. 2018. Effect of seed dressing on germination and root rot of soybean. International Journal of Chemical Studies. 6(1):653-655

Jash, S. and Pan,S. 2007. Variability in antagonistic activity and root colonizing behavior of Trichoderma isolates. Journal of Tropical Agriculture, 95(2):29-35.

Khodke, S.W. and Raut,B.T. 2011. Management of root rot/collar rot of soybean. Indian Phytopathology, 63(3):298-301.

Konde, S.A., R.B. Kothikar and Koch,M. 2017. Effect of different treatments of fungicides and bioagents on collar rot disease of soybean and its grain yield. JInno Agri. 4(1):17-20.

Meher, J., P. Kashyap, S.S. Sonkar, S.N. Singh and Kumari,S. 2018. Studies on native isolates of fungal and bacterial bio-agents against collar rot of Chickpea. Int.J.Curr.Microbiol. App. Sci. 7(1):226-238.

Olive, L.S., D.C. Bain and Lefebvre,C.L. 1945. A leaf spot of cowpea and soybean caused by an undescribed species of Helminthosporium. Phytopathology, 35: 822-831.

Parmar, V., S.N. Singh, G.P. Tiwari andSing,hP. 2018. Relative efficacy of Bio pesticides in managmentof dry root rot and collar rot in soyabean and chickpea. International Journal of Chemical Studies, 6(2):83-86.

Patel, M.P. 2005. Studies on Corynespora cassiicola (Bark. and Curt.) Wei. causing target spot of Soybean (Glycine $\max ($ L.) Merrill)" M.Sc. (Ag.) Thesis, Indira Gandhi Agricultural University Raipur.

Rajendraprasad, M., B.V. Sagar, G.U. Devi and Rao,S.R.K. 2017. Biological control of tomato damping off caused by Sclerotium rolfsii. Journal of Entomology and Zoology Studies, 5(5):113-119.

Rudresh, D.L., M.K. Shivaprakash and Prasad,R.D. 2005. Potential of Trichoderma spp. as bio-control agents of pathogens involved in wilt complex of chickpea (Cicer arietinum L.). Journal of Biological Control, 19(2):157-166.

Sharma, P. and Patel,R.M.R. 2017. Soybean monitor/ market watch. ICAR- Indian Institute of Soybean Research, 9: 1-9.

Singh, S. and Tiwari,R.K.S. 2018. Efficacy of Trichoderma harzianumalone and its integration with fungicides against collar rot caused by Sclerotium rolfsii in Chickpea. International Journal of Agriculture Sciences, 10(9):5945-5947.

Singh, S., V.K. Nirmalkar, R.K.S. Tiwari, A. Jangre and Kumar,P. 2017. Integration of Trichoderma, Pseudomonas and fungicides for the control of collar rot 
disease of chickpea (Cicer arietinum L.). International Journal of Agriculture, Environment and Biotechnology, 10(1):125-131.

Smith, L. 2008. Host range, phylogenetic and pathogenic diversity of Corynespora cassiicola (Berk. \& Curt.) Wei. PhD dissertation, University of Florida, Gainesville.

Sunil, K., C. Gireesh, S. Kumar and Singh, K.K. 2015. Occurrence and progression of some foliar diseases of Soybean [Glycine $\max (\mathrm{L}$.$) Merrill].$
Annals of Plant Protection Sciences, 23(2):308-312.

Suryawanshi, A.P., A.S. Borgaonkar, D.P. Kuldhar and Dey,U. 2015. Integrated management of collar rot (Sclerotium rolfsii) of brinjal (Solanum melongena). Indian Phytopath. 68 (2):189-195.

Swathi, B., A.K. Patibanda andPrasuna,R.P. 2015. Antagonistic efficacy of Trichoderma spp. on Sclerotium rolfsii in vitro. IOSR Journal of Agriculture and Veterinary Science, 8(7):19-22.

\section{How to cite this article:}

Shyam Singh, Anjeet Jangre and Tiwari, R. K. S. 2021. Impacts of Indigenous Trichoderma harzianum, Trichoderma viride and Pseudomonas fluorescens on Microbial Population in Soil, Plant Growth Promoting and Disease Control Potential in Soybean. Int.J.Curr.Microbiol.App.Sci. 10(02): 250-264. doi: https://doi.org/10.20546/ijcmas.2021.1002.031 